\title{
Synthesis of 3-(Arylthio)propionic Acids from Nonactivated Aryl lodides and their Use as Odorless Aryl Mercaptan Surrogates
}

\author{
B. Menczinger \\ A. Nemes \\ A. Csámpai \\ J. Rábai* (D)
}

Eötvös Loránd University, Institute of Chemistry, Pázmány Péter sétány 1/A, 1117 Budapest, Hungary rabai@elte.hu

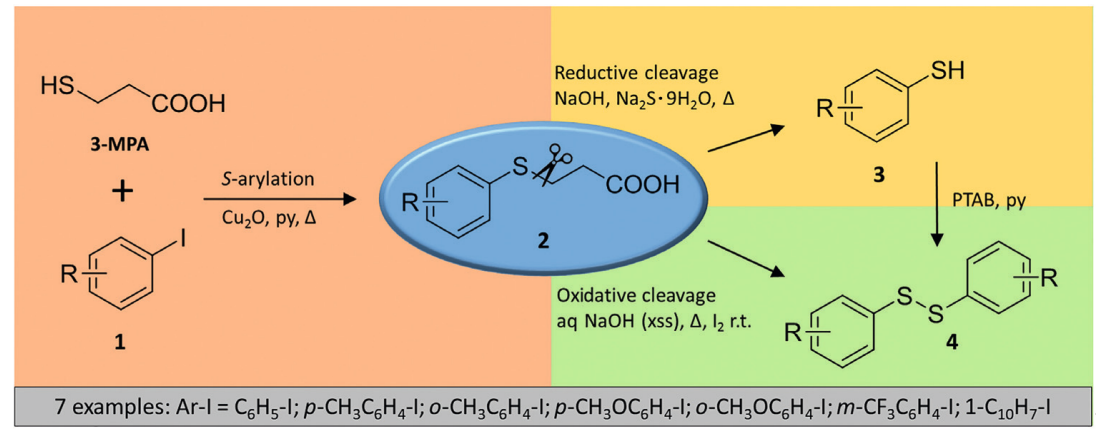

Received: 14.11.2017

Accepted after revision: 10.02.2018

Published online: 14.03 .2018

DOI: 10.1055/s-0036-1591771; Art ID: so-2017-d0056-op

License terms: cc)

Abstract The reaction of aryl iodides, 3-mercaptopropionic acid, and $\mathrm{Cu}_{2} \mathrm{O}$ in refluxing pyridine resulted in the formation of 3-(arylthio)propionic acids in good to excellent yield. The latter 3-(arylthio)propionic acids - as novel aryl mercaptan equivalents - gave aryl mercaptans or diaryl disulfides, respectively, on reductive $\left(\mathrm{Na}_{2} \mathrm{~S}\right)$ or oxidative $\left(\mathrm{I}_{2}\right)$ cleavage in alkaline media. The symmetrical disulfides can also be prepared by oxidizing their precursor mercaptans with phenyltrimethylammoniumtribromide in pyridine at ambient temperature.

Key words 3-mercaptopropionic acid, sulfur-transfer reagent, 3-(arylthio)propionic acid, reverse Michael reaction, copper(I) oxide

3-(Arylthio)propionic acids are important compounds in biochemistry and pharmaceutical chemistry. They are used as sulfur-transfer reagents and building blocks for the synthesis of compound families with diverse biological activity. For example, 5 has anticancer activity, ${ }^{1} \mathbf{6}$ is a monoamine oxidase inhibitor, ${ }^{2} \mathbf{7}$ has antihepatitis effects ${ }^{3}$ and Meniere's disease can be treated with $\mathbf{8}^{4}$ (Figure 1 ). Furthermore, other important active pharmaceutical ingredients, such as (thio)pyranones, piperidones, ${ }^{5}$ and benzodiazepines $^{6}$ are easily synthesized from 3-(arylthio)propionic acid derivatives. 3-(Arylthio)propionic acids have usually been synthesized from their precursor aryl mercaptans by conjugated addition reactions using acrylic acid, acrylic esters or acrylonitrile, ${ }^{7}$ or by the alkylation of thiophenol derivatives using $\beta$-halopropionic acids or esters, or $\beta$-propiolactone. ${ }^{8,9}$ They can also be synthesized by nickel-catalyzed S-arylation of 3-mercaptopropionic acid (3-MPA) with aryl iodides. ${ }^{10}$ On the other hand, methyl 3-(arylthio)propio- nates are accessible by base-induced cleavage of sulfonium salts, prepared by condensation of electron-rich arenes and appropriate sulfoxides. ${ }^{11}$
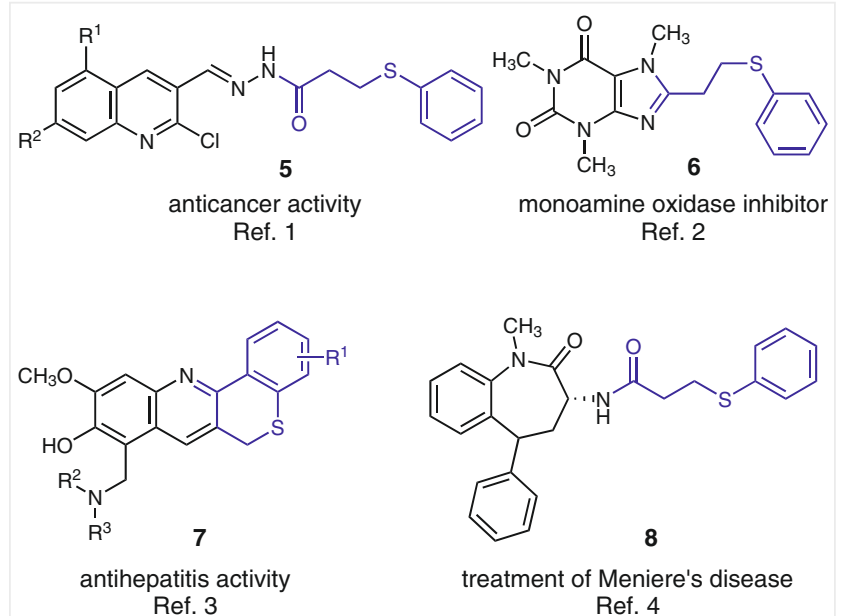

Figure 1 3-(Arylthio)propionic acids as building blocks and transfer reagents in active pharmaceutical ingredients (APIs)

3-(Arylthio)propionic acids are shelf-stable sources of thiols. ${ }^{2}$ Thiols are an important class of compounds because of the special ability of the mercapto group to bind to metals and regulate redox reactions. ${ }^{13}$ Aromatic thiols are often used for the synthesis and stabilization of metal nanoparticles ${ }^{14}$ and for modification of metal or metal oxide surfaces. ${ }^{15}$ Mercaptans also have biological activity, they play an important role in the regulation mechanism of redox systems with biological importance, ${ }^{16}$ and this moiety can be found in anti-HIV and anticancer agents. ${ }^{17}$ Diaryl and dialkyl disulfides are stable sources of thiols ${ }^{18}$ and starting materials of several sulfur-containing reagents, 
such as sulfenic acids, ${ }^{19}$ sulfinic esters, ${ }^{20}$ sulfinyl chlorides, ${ }^{21}$ and thiocarbamates. ${ }^{22}$ They are also used as antitumor $^{23}$ and anti-HIV agents. ${ }^{24}$

Although many procedures are known for the preparation of aryl mercaptans ${ }^{25}$ and diaryl disulfides, ${ }^{26}$ only a few involve the reaction of nonactivated aryl iodides with appropriate sulfur-transfer reagents such as copper(I) thiobenzoate $^{27}$ or copper(I) thiocyanate. ${ }^{26 \mathrm{~b}}$ The latter methods provide some advantages starting from accessible aryl iodides and reagents, but they involve the use of a carcinogenic solvent, HMPT.

In biological systems, thioethers are often used for reversible conjugation of thiols, ${ }^{28}$ where the appropriate thiols are liberated by retro-Michael reaction. This approach is of intense interest for the development of fluorescent probes $^{29}$ and drug-delivery systems. ${ }^{30} 3$-(Arylthio)propionic acids 2 also can be cleaved by retro-Michael reaction to afford the appropriate thiolates $\mathbf{1 1}$ under laboratory conditions. ${ }^{31}$ In our previous work, 3-MPA was used as a sulfurtransfer reagent in the synthesis of symmetric diaryl sulfides (Scheme 1). ${ }^{32}$ We proposed that the reaction went through a 3-(arylthio)propionic acid intermediate 10, which, in the next step, was cleaved to afford the arylthiolate intermediate 11. The thiolate intermediate was isolated only in one specific case, when we adjusted the $\mathrm{pH}$ of the

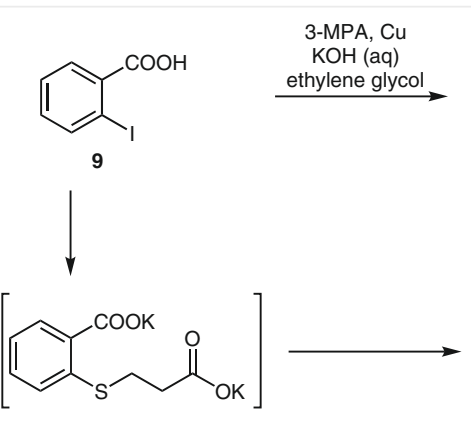

10

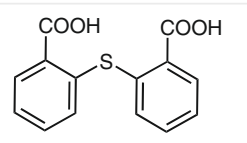

12

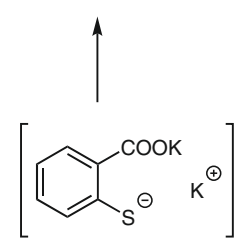

11
Scheme 1 Synthesis of a symmetrical diaryl sulfide via 3-(arylmercapto)propionic acid $\mathbf{1 0}$ and arenethiolate $\mathbf{1 1}$ intermediate (cf. Ref. ${ }^{19}$ ) system to prevent the decomposition of 3-(8-carboxy-1naphthylthio)propionic acid (14) into 8-mercapto-1-naphthalene-carboxylate, which, on acidification, gave the appropriate thiolactone (Scheme 2 ). ${ }^{32}$

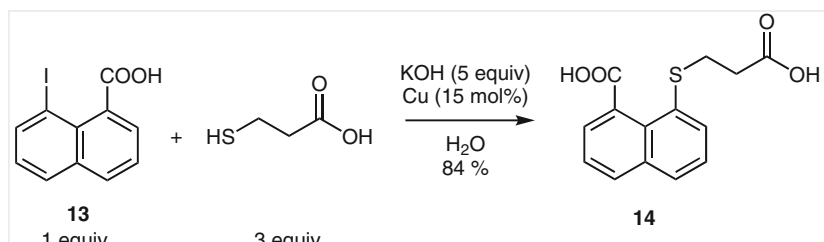

Scheme 2 Synthesis of 3-(8-carboxy-1-naphthylthio)propionic acid

In this study, as opposed to our earlier method allowing the synthesis of symmetrical diaryl sulfides in a one-pot reaction, we modified the reaction conditions to enable the isolation of 3-(arylthio)propionic acids $\mathbf{2}$ as the main products of the reaction of 3-MPA and aryl iodides.

3-(Arylthio)propionic acids 2 can be synthesized by copper-mediated C-S bond formation of 3-mercaptopropionic acid and nonactivated aryl iodides $\mathbf{1}$. These compounds are important intermediates in the synthesis of arylmercaptans 3 and diaryl disulfides 4 (Scheme 3). To find the optimal reaction conditions, the solvent, the amount of $\mathrm{Cu}_{2} \mathrm{O}$, and the reaction time were varied (Table 1$)$.

3-(Phenylthio)propionic acid was synthesized in good yield by using equivalent amounts of iodobenzene, 3-mercaptopropionic acid, and 0.5 equiv of $\mathrm{Cu}_{2} \mathrm{O}$ in refluxing pyridine for $6 \mathrm{~h}$ (Table 1, entry 2). Substituted 3-(arylthio)propionic acids $\mathbf{2 a - g}$ were prepared with good yields (Table 2).

Under alkaline conditions, thioether groups that are in the $\gamma$-position relative to a carbonyl group can be cleaved by retro-Michael reaction. This reaction was first observed by Holmberg and Schjånberg. They reported that diphenyl disulfide and 3-hydroxypropionic acid are formed when an aqueous $\mathrm{NaOH}$ solution of 3-(phenylthio)propionic acid was exposed to air, but no formation of dibenzyl disulfide was observed under the same conditions using 3-(benzylthio)propionic acid. ${ }^{31}$ These observations can be interpreted by considering the higher acidity of thiophenol

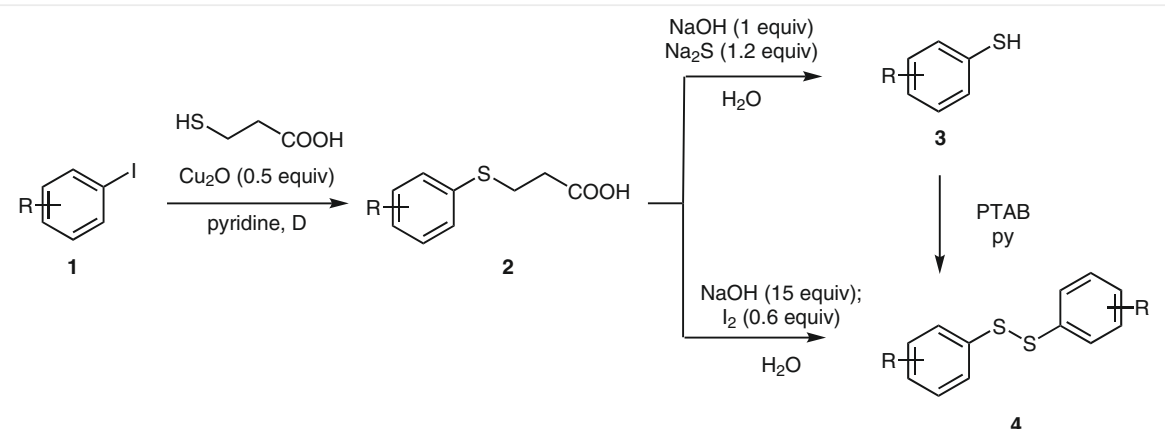

Scheme 3 Synthesis of arylmercaptans and diaryl disulfides via 3-arylmercaptopropionic acid intermediate 
Table 1 Optimization of the Synthesis of 3-(Phenylthio)propionic Acid

\begin{tabular}{lllll}
\multicolumn{1}{c}{} & & & \\
\hline Entry & $\mathrm{Cu}_{2} \mathrm{O}$ (equiv.) & Solvent & Reaction time (h) & Yield (\%) \\
\hline 1 & 0.5 & py & 3 & 54 \\
2 & 0.5 & py & 6 & 67 \\
3 & 0.5 & py & 12 & 52 \\
4 & 0.5 & DMF & 3 & 48 \\
5 & 0.5 & DMF+ 4 eq & 3 & 47 \\
6 & 1.0 & py & 3 & 14 \\
7 & 0.05 & py & 3 & 0 \\
\hline
\end{tabular}

$\left(\mathrm{p} K_{\mathrm{a}} 6.52\right)$ when compared to benzyl mercaptan ( $\left.\mathrm{p} K_{\mathrm{a}} 9.43\right)$, thus the former is a better leaving group than the latter. In a control experiment we found that 3-(n-butylthio)propionic acid $\left(n-\mathrm{C}_{4} \mathrm{H}_{9} \mathrm{~S}-\mathrm{CH}_{2} \mathrm{CH}_{2} \mathrm{CO}_{2} \mathrm{H}\right)$ was also stable to aq-NaOH in the presence of air (cf. $n$-butyl mercaptan, $\mathrm{p} K_{\mathrm{a}} 10.66$ ). ${ }^{35}$ Retro-Michael reaction also took place at neutral $\mathrm{pH}$, promoted by excess thiol. ${ }^{29}$ In this case, the formed acrylic acid is quenched, thus the equilibrium is shifted towards the cleavage reaction. Acrylic acid also reacts in situ with sulfide $^{36}$ or hydroxide nucleophiles. On the other hand, the equilibrium can be shifted by oxidation of the formed mercaptides.

Table 2 Synthesis of 3-(Arylthio)propionic Acids

\begin{tabular}{|c|c|c|c|c|}
\hline & & & \multicolumn{2}{|c|}{$2 a-g$} \\
\hline Entry & $\mathrm{R}$ & Product & Yield (\%) & Lit. $^{a}$ \\
\hline 1 & $\mathrm{H}$ & $2 a$ & 67 & $7 a, 8 c$ \\
\hline 2 & $4-\mathrm{CH}_{3}$ & $2 b$ & 77 & $8 b$ \\
\hline 3 & $2-\mathrm{CH}_{3}$ & $2 c$ & 68 & 9 \\
\hline 4 & $4-\mathrm{OCH}_{3}$ & $2 d$ & 69 & $7 a, 33$ \\
\hline 5 & $2-\mathrm{OCH}_{3}$ & $2 e$ & 66 & $7 \mathrm{a}, 8 \mathrm{~d}$ \\
\hline 6 & $3-\mathrm{CF}_{3}$ & $2 f$ & 59 & 34 \\
\hline 7 & 1-naphthyl & $2 g$ & 51 & $8 \mathrm{~b}$ \\
\hline
\end{tabular}

a The same product was synthesized by a different method.

In our first set of experiments, 3-(arylthio)propionic acids $\mathbf{2 a}-\mathbf{g}$ were reacted with sodium hydroxide in the presence of excess sodium sulfide under nitrogen atmosphere.
Arylmercaptans 3a-g were isolated from the reaction mixture in good to excellent yield (Table 3). The synthesis of 4methylbenzenethiol (3b) was reproduced in $\mathrm{D}_{2} \mathrm{O}$. In this case, the retro-Michael reaction took place in $2 \mathrm{~h}$. The acrylic acid concentration was low during the reaction, since the formed acrylic acid reacted with the excess sodium sulfide present in the reaction mixture (Figure 2). ${ }^{13} \mathrm{C}$ NMR spectrum of the isolated byproduct is consistent with the structure of the disodium salt of 3-mercaptopropionic acid (3MPA).

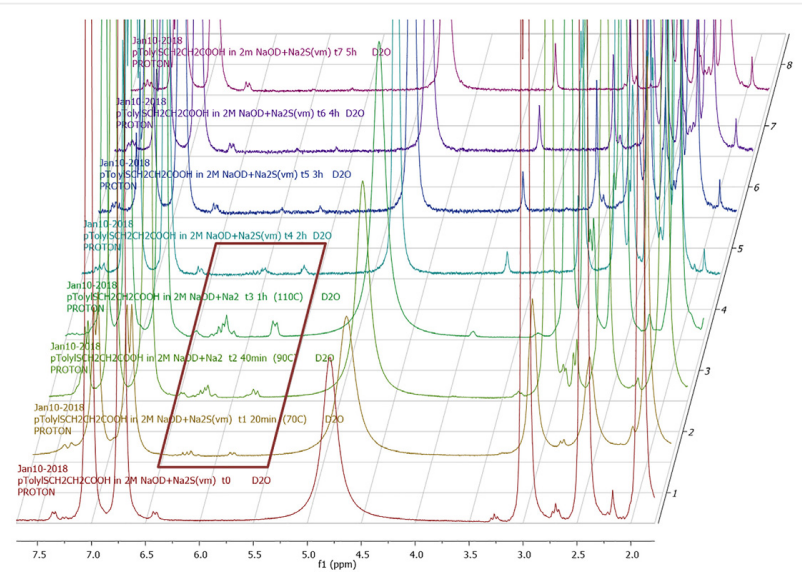

Figure $2{ }^{1} \mathrm{H}$ NMR spectra of the reaction mixture of the synthesis of $\mathbf{3 b}$

In the second set of experiments, arylmercaptans $\mathbf{3}$ formed in the retro-Michael reaction were oxidized in situ by iodine ${ }^{37}$ to afford diaryl disulfides $\mathbf{4 a -} \mathbf{g}$ in good yield (Table 4).

We have found that mercaptans $3 \mathbf{3 a - g}$ could also be oxidized to symmetrical disulfides $\mathbf{4 a - g}$ in high yields under homogeneous conditions in pyridine with phenyltrimethylammonium tribromide (PTAB) at room temperature (Table 5).

In addition, the stench originating from minute amounts of aryl mercaptans in the glassware can be eliminated quickly by rinsing them with a few milliliters of PTAB/pyridine solution because of their oxidation to less volatile and odorous disulfides. This shelf-stable PTAB reagent has been used for the selective oxidation of sulfides to sulfoxides. ${ }^{42}$

In conclusion, arylmercaptans and diaryl disulfides were synthesized via 3-(arylmercapto)propionic acid intermediates in good yields. The availability of aryl iodides and reagents used, coupled with easy product isolation, make these synthetic methods attractive. 
Table 3 Synthesis of Arylmercaptans

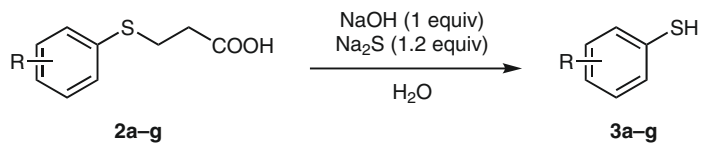

\begin{tabular}{llll}
\hline Entry & $\mathrm{R}$ & Product & Yield (\%) \\
\hline 1 & $\mathrm{H}$ & $\mathbf{3 a}$ & 65 \\
2 & $4-\mathrm{CH}_{3}$ & $\mathbf{3 b}$ & 78 \\
3 & $2-\mathrm{CH}_{3}$ & $\mathbf{3 c}$ & 99 \\
4 & $4-\mathrm{OCH}_{3}$ & $\mathbf{3 d}$ & 97 \\
5 & $2-\mathrm{OCH}_{3}$ & $\mathbf{3 e}$ & 100 \\
6 & $3-\mathrm{CF}_{3}$ & $\mathbf{3 f}$ & 81 \\
7 & $1-$ naphthyl & $\mathbf{3 g}$ & 92 \\
\hline
\end{tabular}

Table 4 Synthesis of Diaryl Disulfides from 3-(Arylthio)propionic Acids

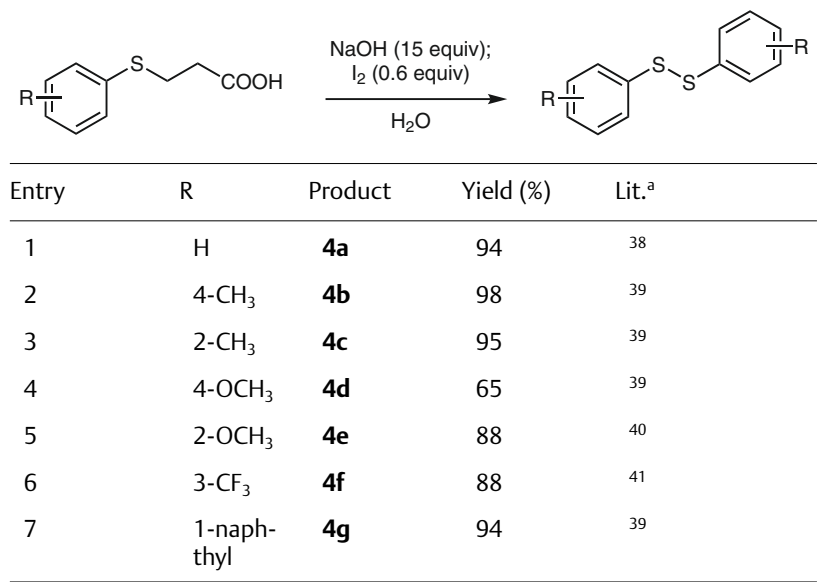

a The same product was synthesized by a different method.

Table 5 Synthesis of Diaryldisulfides by Oxidizing Thiols

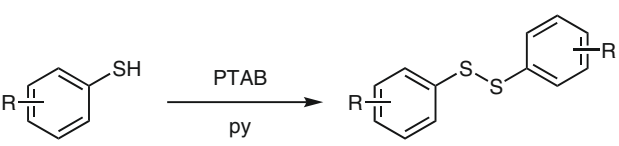

\begin{tabular}{llll}
\hline Entry & $\mathrm{R}$ & Product & Yield (\%) \\
\hline 1 & $\mathrm{H}$ & $\mathbf{4 a}$ & 88 \\
2 & $4-\mathrm{CH}_{3}$ & $\mathbf{4 b}$ & 53 \\
3 & $2-\mathrm{CH}_{3}$ & $\mathbf{4 c}$ & 61 \\
4 & $4-\mathrm{OCH}_{3}$ & $\mathbf{4 d}$ & 78 \\
5 & $2-\mathrm{OCH}_{3}$ & $\mathbf{4 e}$ & 63 \\
6 & $3-\mathrm{CF}_{3}$ & $\mathbf{4 f}$ & 56 \\
7 & $1-\mathrm{naphthyl}$ & $\mathbf{4 g}$ & 53 \\
\hline
\end{tabular}

${ }^{1} \mathrm{H}$ and ${ }^{13} \mathrm{C}$ NMR spectra were recorded with a Bruker Avance 250 $\mathrm{MHz}$ instrument using a $5 \mathrm{~mm}{ }^{1} \mathrm{H}$ - and BB-channel probe head at r.t. $(295 \pm 2 \mathrm{~K})$ in $\mathrm{CDCl}_{3}$. Chemical shifts $(\delta)$ are given in ppm units relative to the internal standards: TMS $\left(\delta=0.00 \mathrm{ppm}\right.$ for $\left.{ }^{1} \mathrm{H}\right)$. Melting points were determined with a Boetius micro melting point apparatus and are uncorrected.

\section{3-(8-Carboxy-1-naphthylthio)propionic Acid (14) $)^{32}$}

A mixture of 3-mercaptopropionic acid (3-MPA) (5.29 mL, 60.7 $\mathrm{mmol}$ ), $\mathrm{KOH}$ (5.68 g, $101 \mathrm{mmol}$ ) in water (16 mL), 8-iodo-1-naphthoic acid $(6.0 \mathrm{~g}, 20.1 \mathrm{mmol})$ and $\mathrm{Cu}$ powder $(0.2 \mathrm{~g}, 3.15 \mathrm{mmol})$ was stirred and heated at reflux for $5 \mathrm{~h}$ under Ar. Then the mixture was diluted with water $(80 \mathrm{~mL})$, filtered through Celite, and the filtrate was acidified with $6 \mathrm{M} \mathrm{HCl}$ to $\mathrm{pH} 1$. The pale-yellow precipitate was filtered and washed with cold water. Then it was dissolved in aq- $\mathrm{KHCO}_{3}$, filtered and acidified with $6 \mathrm{M} \mathrm{HCl}$ to $\mathrm{pH} 1$, and dried in a dessicator over $\mathrm{KOH}$ pellets.

Yield: $4.58 \mathrm{~g}$ (16.9 mmol, 84\%); white needles; $\mathrm{mp} 158-159{ }^{\circ} \mathrm{C}$. The physical and spectral properties of the product are identical with those reported. ${ }^{19}$

\section{Preparation of 3-(Arylthio)propionic Acids (2a-g); General Proce- dure}

A mixture of 3-MPA ( $8.7 \mathrm{~mL}, 100 \mathrm{mmol}), \mathrm{Cu}_{2} \mathrm{O}(7.15 \mathrm{~g}, 50 \mathrm{mmol})$, and aryl iodide $(100 \mathrm{mmol})$ in absolute pyridine $(80 \mathrm{~mL})$ was heated and stirred at $120-130{ }^{\circ} \mathrm{C}$ under $\mathrm{N}_{2}$ atmosphere for $6 \mathrm{~h}$. The solvent was then evaporated in vacuo, $6 \mathrm{M} \mathrm{HCl}(80 \mathrm{~mL})$ was added to the residue and the mixture was stirred for $30 \mathrm{~min}$ at $90{ }^{\circ} \mathrm{C}$. The reaction mixture was cooled to r.t., filtered, washed with water $(3 \times 20 \mathrm{~mL})$ and dried over $\mathrm{cc}_{2} \mathrm{SO}_{4}$. The anhydrous solid material was extracted with boiling acetone $(3 \times 100 \mathrm{~mL})$, and the combined filtrates were evaporated under reduced pressure. $1 \mathrm{M} \mathrm{KHCO}_{3}(120 \mathrm{~mL})$ was added to the residue and the unreacted aryl iodide was steam-distilled from the mixture. Charcoal was added to the residue and the mixture was stirred for $5 \mathrm{~min}$. The filtered solution was acidified with $6 \mathrm{M} \mathrm{HCl}$ to $\mathrm{pH} 1$. The precipitate was filtered, washed with water $(20 \mathrm{~mL})$ and dried over $\mathrm{P}_{2} \mathrm{O}_{5}$. This product was used in the next reaction step without further purification or recrystallized to afford pure samples.

\section{3-(Phenylthio)propionic Acid (2a) ${ }^{7 a}$}

3-MPA (8.7 mL, $10.61 \mathrm{~g}, 100 \mathrm{mmol})$ was reacted with $\mathrm{Cu}_{2} \mathrm{O}(7.15 \mathrm{~g}, 50$ $\mathrm{mmol}$ ) and iodobenzene $1 \mathrm{a}(11.2 \mathrm{~mL}, 20.40 \mathrm{~g}, 100 \mathrm{mmol})$.

Yield: $12.21 \mathrm{~g}$ (67\%); white crystals; $\mathrm{mp} 60-61{ }^{\circ} \mathrm{C}\left(\mathrm{EtOH}-\mathrm{H}_{2} \mathrm{O}\right)$ as reported

${ }^{1} \mathrm{H}$ NMR $\left(250 \mathrm{MHz}, \mathrm{CDCl}_{3}\right): \delta=11.52\left(\mathrm{~s}, 1 \mathrm{H}, \mathrm{SCH}_{2} \mathrm{CH}_{2} \mathrm{COOH}\right), 7.50-$ $7.10(\mathrm{~m}, 5 \mathrm{H}, \mathrm{Ar}-\mathrm{H}), 3.15\left(\mathrm{t},{ }^{3} \mathrm{~J}_{\mathrm{H}-\mathrm{H}}=7.5 \mathrm{~Hz}, 2 \mathrm{H}, \mathrm{SCH}_{2} \mathrm{CH}_{2} \mathrm{COOH}\right), 2.64(\mathrm{t}$, $\left.{ }^{3} J_{\mathrm{H}-\mathrm{H}}=7.5 \mathrm{~Hz}, 2 \mathrm{H}, \mathrm{SCH}_{2} \mathrm{CH}_{2} \mathrm{COOH}\right)$.

${ }^{13} \mathrm{C}$ NMR $\left(75 \mathrm{MHz}, \mathrm{CDCl}_{3}\right): \delta=178.6\left(\mathrm{SCH}_{2} \mathrm{CH}_{2} \mathrm{COOH}\right), 135.3(\mathrm{Ar}-\mathrm{C} 1)$, 130.7 (Ar-C2), 129.5 (Ar-C3), 127.1 (Ar-C4), $34.6\left(\mathrm{SCH}_{2} \mathrm{CH}_{2} \mathrm{COOH}\right)$, $29.2\left(\mathrm{SCH}_{2} \mathrm{CH}_{2} \mathrm{COOH}\right)$.

\section{3-(4-Tolylthio)propionic Acid (2b) (b $^{\text {b }}$}

3-MPA ( $8.7 \mathrm{~mL}, 10.61 \mathrm{~g}, 100 \mathrm{mmol}$ ) was reacted with $\mathrm{Cu}_{2} \mathrm{O}$ (7.15 g, 50 $\mathrm{mmol})$ and 4-iodotoluene $\mathbf{1 b}(21.80 \mathrm{~g}, 100 \mathrm{mmol})$.

Yield: $15.11 \mathrm{~g}$ (77\%); white crystals; $\mathrm{mp} 71-72{ }^{\circ} \mathrm{C}$ (ligroin). ${ }^{1} \mathrm{H}$ NMR $\left(250 \mathrm{MHz}, \mathrm{CDCl}_{3}\right): \delta=11.37\left(\mathrm{~s}, 1 \mathrm{H}, \mathrm{SCH}_{2} \mathrm{CH}_{2} \mathrm{COOH}\right), 7.29(\mathrm{~d}$, $\left.{ }^{3} J_{\mathrm{H}-\mathrm{H}}=8.3 \mathrm{~Hz}, 2 \mathrm{H}, \mathrm{Ar}-\mathrm{H} 2\right), 7.11\left(\mathrm{~d},{ }^{3} \mathrm{~J}_{\mathrm{H}-\mathrm{H}}=8.3 \mathrm{~Hz}, 2 \mathrm{H}, \mathrm{Ar}-\mathrm{H} 3\right), 3.09(\mathrm{t}$, $\left.{ }^{3} J_{\mathrm{H}-\mathrm{H}}=7.5 \mathrm{~Hz}, 2 \mathrm{H}, \quad \mathrm{SCH}_{2} \mathrm{CH}_{2} \mathrm{COOH}\right), 2.60\left(\mathrm{t}, \quad 3_{\mathrm{H}-\mathrm{H}}=7.5 \mathrm{~Hz}, 2 \mathrm{H}\right.$, $\left.\mathrm{SCH}_{2} \mathrm{CH}_{2} \mathrm{COOH}\right) ; 2.28\left(\mathrm{~s}, 3 \mathrm{H}, \mathrm{CH}_{3}\right)$. 
${ }^{13} \mathrm{C}$ NMR (75 MHz, $\left.\mathrm{CDCl}_{3}\right): \delta=178.7\left(\mathrm{SCH}_{2} \mathrm{CH}_{2} \mathrm{COOH}\right), 137.5(\mathrm{Ar}-\mathrm{C} 4)$, 131.7 (Ar-C3), 131.4 (Ar-C1), 130.2 (Ar-C2), $34.7\left(\mathrm{SCH}_{2} \mathrm{CH}_{2} \mathrm{COOH}\right.$ ), $29.9\left(\mathrm{SCH}_{2} \mathrm{CH}_{2} \mathrm{COOH}\right), 21.4\left(\mathrm{CH}_{3}\right)$.

\section{3-(2-Tolylthio)propionic Acid (2c) ${ }^{9}$}

3-MPA (8.7 mL, $10.61 \mathrm{~g}, 100 \mathrm{mmol}$ ) was reacted with $\mathrm{Cu}_{2} \mathrm{O}$ (7.15 g, 50 $\mathrm{mmol}$ ) and 2-iodotoluene $1 \mathrm{c}(12.73 \mathrm{~mL}, 21.80 \mathrm{~g}, 100 \mathrm{mmol})$.

Yield: $13.35 \mathrm{~g}$ (68\%) white crystals, $\mathrm{mp} 93-94{ }^{\circ} \mathrm{C}\left(\mathrm{C}_{6} \mathrm{H}_{6}\right.$-hexane).

${ }^{1} \mathrm{H}$ NMR (250 MHz, $\left.\mathrm{CDCl}_{3}\right): \delta=11.11\left(\mathrm{~s}, 1 \mathrm{H}, \mathrm{SCH}_{2} \mathrm{CH}_{2} \mathrm{COOH}\right), 7.45-$ $7.05(\mathrm{~m}, 4 \mathrm{H}, \mathrm{ArH}), 3.12\left(\mathrm{t}, 3_{\mathrm{H}-\mathrm{H}}=7.5 \mathrm{~Hz}, 2 \mathrm{H}, \mathrm{SCH}_{2} \mathrm{CH}_{2} \mathrm{COOH}\right), 2.64(\mathrm{t}$, $\left.{ }^{3} J_{\mathrm{H}-\mathrm{H}}=7.5 \mathrm{~Hz}, 2 \mathrm{H}, \mathrm{SCH}_{2} \mathrm{CH}_{2} \mathrm{COOH}\right), 2.37\left(\mathrm{~s}, 3 \mathrm{H}, \mathrm{CH}_{3}\right)$.

${ }^{13} \mathrm{C}$ NMR (75 MHz, $\left.\mathrm{CDCl}_{3}\right): \delta=178.3\left(\mathrm{SCH}_{2} \mathrm{CH}_{2} \mathrm{COOH}\right), 139.0(\mathrm{Ar}-\mathrm{C} 2)$, 134.6 (Ar-C1), 130.7 (Ar-C5), 129.7 (Ar-C6), 126.9 (Ar-C4,C3), 34.4 $\left(\mathrm{SCH}_{2} \mathrm{CH}_{2} \mathrm{COOH}\right), 28.3\left(\mathrm{SCH}_{2} \mathrm{CH}_{2} \mathrm{COOH}\right), 20.8\left(\mathrm{CH}_{3}\right)$.

\section{3-((4-Methoxyphenyl)thio)propionic Acid (2d) $)^{\text {7a }}$}

3-MPA ( $8.7 \mathrm{~mL}, 10.61 \mathrm{~g}, 100 \mathrm{mmol}$ ) was reacted with $\mathrm{Cu}_{2} \mathrm{O}$ (7.15 g, 50 $\mathrm{mmol}$ ) and 4-iodoanisole $1 \mathrm{~d}(23.40 \mathrm{~g}, 100 \mathrm{mmol})$.

Yield: $14.64 \mathrm{~g}$ (69\%); white crystals; $\mathrm{mp} 81-82{ }^{\circ} \mathrm{C}\left(\mathrm{C}_{6} \mathrm{H}_{6}\right.$-petrol ether). ${ }^{1} \mathrm{H}$ NMR $\left(250 \mathrm{MHz}, \mathrm{CDCl}_{3}\right): \delta=11.26\left(\mathrm{~s}, 1 \mathrm{H}, \mathrm{SCH}_{2} \mathrm{CH}_{2} \mathrm{COOH}\right), 7.36$ (d, $\left.{ }^{3} J_{\mathrm{H}-\mathrm{H}}=9.0 \mathrm{~Hz}, 2 \mathrm{H}, \mathrm{Ar}-\mathrm{H} 3\right), 6.82\left(\mathrm{~d},{ }^{3} J_{\mathrm{H}-\mathrm{H}}=9.0 \mathrm{~Hz}, 2 \mathrm{H}, \mathrm{Ar}-\mathrm{H} 2\right), 3.76(\mathrm{~s}$, $\left.3 \mathrm{H}, \mathrm{CH}_{3}\right), 3.01\left(\mathrm{t}, 3_{\mathrm{H}-\mathrm{H}}=7.5 \mathrm{~Hz}, 2 \mathrm{H}, \mathrm{SCH}_{2} \mathrm{CH}_{2} \mathrm{COOH}\right), 2.58\left(\mathrm{t}, 3_{\mathrm{H}-\mathrm{H}}=\right.$ $\left.7.5 \mathrm{~Hz}, 2 \mathrm{H}, \mathrm{SCH}_{2} \mathrm{CH}_{2} \mathrm{COOH}\right)$.

${ }^{13} \mathrm{C}$ NMR (75 MHz, $\left.\mathrm{CDCl}_{3}\right): \delta=178.6\left(\mathrm{SCH}_{2} \mathrm{CH}_{2} \mathrm{COOH}\right), 159.9(\mathrm{Ar}-\mathrm{C} 4)$, 134.8 (Ar-C1), 125.2 (Ar-C2), 115.1 (Ar-C3), $55.7\left(\mathrm{CH}_{3}\right), 34.8$ $\left(\mathrm{SCH}_{2} \mathrm{CH}_{2} \mathrm{COOH}\right), 31.2\left(\mathrm{SCH}_{2} \mathrm{CH}_{2} \mathrm{COOH}\right)$.

\section{3-((2-Methoxyphenyl)thio)propionic Acid (2e) $)^{7 \mathrm{a}}$}

3-MPA (8.7 mL, $10.61 \mathrm{~g}, 100 \mathrm{mmol}$ ) was reacted with $\mathrm{Cu}_{2} \mathrm{O}$ (7.15 g, 50 $\mathrm{mmol}$ ) and 2-iodoanisole $1 \mathrm{e}(13.0 \mathrm{~mL}, 23.40 \mathrm{~g}, 100 \mathrm{mmol})$.

Yield: $14.01 \mathrm{~g}$ (66\%); pale-yellow crystals; $\mathrm{mp} 89^{\circ} \mathrm{C}$.

${ }^{1} \mathrm{H}$ NMR (250 MHz, $\mathrm{CDCl}_{3}$ ): $\delta=11.47$ (s, $1 \mathrm{H}, \mathrm{SCH}_{2} \mathrm{CH}_{2} \mathrm{COOH}$ ), 7.40$7.10(\mathrm{~m}, 3 \mathrm{H}, \mathrm{Ar}-\mathrm{H} 3, \mathrm{H} 4, \mathrm{H6}), 6.88\left(\mathrm{dt},{ }^{3} J_{\mathrm{H}-\mathrm{H}}=7.5 \mathrm{~Hz},{ }^{4} J_{\mathrm{H}-\mathrm{H}}=1.7 \mathrm{~Hz}, 1 \mathrm{H}\right.$, Ar-H5), 3.83 (s, $3 \mathrm{H}, \mathrm{CH}_{3}$ ), $3.09\left(\mathrm{t},{ }^{3} \mathrm{~J}_{\mathrm{H}-\mathrm{H}}=7.5 \mathrm{~Hz}, 2 \mathrm{H}, \mathrm{SCH}_{2} \mathrm{CH}_{2} \mathrm{COOH}\right.$ ), $2.62\left(\mathrm{t},{ }^{3} \mathrm{H}_{\mathrm{H}-\mathrm{H}}=7.5 \mathrm{~Hz}, 2 \mathrm{H}, \mathrm{SCH}_{2} \mathrm{CH}_{2} \mathrm{COOH}\right)$.

${ }^{13} \mathrm{C}$ NMR $\left(75 \mathrm{MHz}, \mathrm{CDCl}_{3}\right): \delta=178.6\left(\mathrm{SCH}_{2} \mathrm{CH}_{2} \mathrm{COOH}\right), 158.5(\mathrm{Ar}-\mathrm{C} 2)$, 131.8 (Ar-C6), 128.7 (Ar-C4), 123.0 (Ar-C1), 121.5 (Ar-C3), 111.2 (ArC5), $56.2\left(\mathrm{CH}_{3}\right), 34.7\left(\mathrm{SCH}_{2} \mathrm{CH}_{2} \mathrm{COOH}\right), 27.5\left(\mathrm{SCH}_{2} \mathrm{CH}_{2} \mathrm{COOH}\right)$.

\section{3-((3-(Trifluoromethyl)phenyl)thio)propionic Acid (2f) $)^{34}$}

3-MPA (8.7 mL, $10.61 \mathrm{~g}, 100 \mathrm{mmol})$ was reacted with $\mathrm{Cu}_{2} \mathrm{O}(7.15 \mathrm{~g}, 50$ $\mathrm{mmol})$ and 3-(trifluoromethyl)iodobenzene $\mathbf{1 f}(27.20 \mathrm{~g}, 100 \mathrm{mmol})$. Yield: $14.76 \mathrm{~g}(59 \%)$; crystalline material; $\mathrm{mp} 56{ }^{\circ} \mathrm{C}$.

${ }^{1} \mathrm{H}$ NMR $\left(250 \mathrm{MHz}, \mathrm{CDCl}_{3}\right): \delta=11.24\left(\mathrm{~s}, 1 \mathrm{H}, \mathrm{SCH}_{2} \mathrm{CH}_{2} \mathrm{COOH}\right)$, 7.65$7.30(\mathrm{~m}, 4 \mathrm{H}, \mathrm{ArH}), 3.19\left(\mathrm{t}, 3_{\mathrm{H}-\mathrm{H}}=7.5 \mathrm{~Hz}, 2 \mathrm{H}, \mathrm{SCH}_{2} \mathrm{CH}_{2} \mathrm{COOH}\right), 2.63(\mathrm{t}$, $\left.{ }^{3} J_{\mathrm{H}-\mathrm{H}}=7.5 \mathrm{~Hz}, 2 \mathrm{H}, \mathrm{SCH}_{2} \mathrm{CH}_{2} \mathrm{COOH}\right)$.

${ }^{13} \mathrm{C}$ NMR $\left(75 \mathrm{MHz}, \mathrm{CDCl}_{3}\right): \delta=178.5\left(\mathrm{SCH}_{2} \mathrm{CH}_{2} \mathrm{COOH}\right), 137.2(\mathrm{Ar}-\mathrm{C} 1)$, 133.1 (Ar-C5), 131.9 (q, $\left.{ }^{2} J_{\mathrm{C}-\mathrm{F}}=32.5 \mathrm{~Hz}, \mathrm{Ar}-\mathrm{C} 3\right), 129.8$ (Ar-C6), 126.5 (q, $\left.{ }^{3} J_{\mathrm{C}-\mathrm{F}}=3.7 \mathrm{~Hz}, \mathrm{Ar}-\mathrm{C} 2\right), 125.3\left(\mathrm{q},{ }^{1} J_{\mathrm{C}-\mathrm{F}}=272.5 \mathrm{~Hz}, \mathrm{CF}_{3}\right), 123.6\left(\mathrm{q},{ }^{3} J_{\mathrm{C}-\mathrm{F}}=\right.$ 3.7 Hz, Ar-C4), $34.4\left(\mathrm{SCH}_{2} \mathrm{CH}_{2} \mathrm{COOH}\right), 28.7\left(\mathrm{SCH}_{2} \mathrm{CH}_{2} \mathrm{COOH}\right)$.

\section{3-((Naphthalen-1-yl)thio)propionic Acid (2g) ${ }^{8 b}$}

3-MPA (8.7 mL, $10.61 \mathrm{~g}, 100 \mathrm{mmol}$ ) was reacted with $\mathrm{Cu}_{2} \mathrm{O}$ (7.15 g, 50 $\mathrm{mmol}$ ) and 1-iodonaphthalene $1 \mathrm{~g}$ (14.6 mL, $25.41 \mathrm{~g}, 100 \mathrm{mmol})$.

Yield: $11.85 \mathrm{~g}$ (51\%); crystalline material; $\mathrm{mp} 89-90{ }^{\circ} \mathrm{C}$.
${ }^{1} \mathrm{H}$ NMR $\left(250 \mathrm{MHz}, \mathrm{CDCl}_{3}\right): \delta=10.85\left(\mathrm{~s}, 1 \mathrm{H}, \mathrm{SCH}_{2} \mathrm{CH}_{2} \mathrm{COOH}\right), 8.00-$ $7.25(\mathrm{~m}, 6 \mathrm{H}, \mathrm{ArH}), 8.43\left(\mathrm{dd},{ }^{3} J_{\mathrm{H}-\mathrm{H}}=6.5 \mathrm{~Hz},{ }^{4} J_{\mathrm{H}-\mathrm{H}}=2.0 \mathrm{~Hz}, 1 \mathrm{H}, \mathrm{Ar}-\mathrm{H} 8\right)$, $3.17\left(\mathrm{t},{ }^{3} J_{\mathrm{H}-\mathrm{H}}=7.5 \mathrm{~Hz}, 2 \mathrm{H}, \mathrm{SCH}_{2} \mathrm{CH}_{2} \mathrm{COOH}\right), 2.62\left(\mathrm{t},{ }^{3} \mathrm{~J}_{\mathrm{H}-\mathrm{H}}=7.5 \mathrm{~Hz}, 2 \mathrm{H}\right.$, $\left.\mathrm{SCH}_{2} \mathrm{CH}_{2} \mathrm{COOH}\right)$.

${ }^{13} \mathrm{C}$ NMR (75 MHz, $\left.\mathrm{CDCl}_{3}\right): \delta=178.9\left(\mathrm{SCH}_{2} \mathrm{CH}_{2} \mathrm{COOH}\right), 134.5$ (Ar-C9), 133.8 (Ar-C10), 132.3 (Ar-C5), 130.6 (Ar-C1), 129.0 (Ar-C6), 128.7 (ArC2), 127.1 (Ar-C7), 126.7 (Ar-C4), 125.9 (Ar-C8), 125.6 (Ar-C3), 34.2 ( $\left.\mathrm{SCH}_{2} \mathrm{CH}_{2} \mathrm{COOH}\right), 29.5\left(\mathrm{SCH}_{2} \mathrm{CH}_{2} \mathrm{COOH}\right)$.

\section{Preparation of Arylmercaptans (3a-g); General Procedure}

To a solution of 3-(arylthio)propionic acid $(100 \mathrm{mmol})$ in $2 \mathrm{M} \mathrm{NaOH}$ $(50 \mathrm{~mL}) \mathrm{Na}_{2} \mathrm{~S} \cdot 10 \mathrm{H}_{2} \mathrm{O}(28.8 \mathrm{~g}, 120 \mathrm{mmol})$ was added, then the mixture was heated at reflux under $\mathrm{N}_{2}$ atmosphere for $5 \mathrm{~h}$. The solution was cooled to r.t., water $(100 \mathrm{~mL})$ was added and the mixture was acidified with $6 \mathrm{M} \mathrm{HCl}$ to $\mathrm{pH} 2$. The mixture was extracted with $\mathrm{CHCl}_{3}(3 \times 100$ $\mathrm{mL})$, the combined organic phases were washed with $5 \% \mathrm{NaHCO}_{3}$ $(2 \times 50 \mathrm{~mL})$ and dried over $\mathrm{MgSO}_{4}$. The solvent was evaporated and the crude product was purified by distillation under $\mathrm{N}_{2}$. All products 3a-g showed higher than $98 \%$ assay as determined by iodometric $\mathrm{SH}$ titration.

\section{Thiophenol (3a) $)^{43}$}

Compound 2a (18.22 g, $100 \mathrm{mmol}$ ) was reacted with $\mathrm{Na}_{2} \mathrm{~S} \cdot 10 \mathrm{H}_{2} \mathrm{O}$ (28.8 g, $120 \mathrm{mmol})$.

Yield: $7.16 \mathrm{~g}$ (65\%); colorless liquid; bp $169{ }^{\circ} \mathrm{C}$.

${ }^{1} \mathrm{H}$ NMR (250 MHz, $\mathrm{CDCl}_{3}$ ): $\delta=7.3-6.9(\mathrm{~m}, 5 \mathrm{H}, \mathrm{ArH}), 3.34(\mathrm{~s}, 1 \mathrm{H}, \mathrm{SH})$. ${ }^{13} \mathrm{C} \mathrm{NMR}\left(75 \mathrm{MHz}, \mathrm{CDCl}_{3}\right.$ ): $\delta=130.8$ (Ar-C1), 129.3 (Ar-C3), $128.9(\mathrm{Ar}-$ C2), 125.5 (Ar-C4).

\section{4-Methylbenzenethiol (3b) ${ }^{44}$}

Compound 2b (19.93 g, $100 \mathrm{mmol}$ ) was reacted with $\mathrm{Na}_{2} \mathrm{~S} \cdot 10 \mathrm{H}_{2} \mathrm{O}$ (28.8 g, $120 \mathrm{mmol})$.

Yield: $9.69 \mathrm{~g}$ (78\%); crystalline material; $\mathrm{mp} 41-43^{\circ} \mathrm{C}$.

${ }^{1} \mathrm{H} \mathrm{NMR}\left(250 \mathrm{MHz}, \mathrm{CDCl}_{3}\right): \delta=7.19\left(\mathrm{~d},{ }^{3} J_{\mathrm{H}-\mathrm{H}}=8.3 \mathrm{~Hz}, 2 \mathrm{H}, \mathrm{Ar}-\mathrm{H} 2\right), 7.06$ (d, $\left.{ }^{3} J_{\mathrm{H}-\mathrm{H}}=8.3 \mathrm{~Hz}, 2 \mathrm{H}, \mathrm{Ar}-\mathrm{H} 3\right), 3.36(\mathrm{~s}, 1 \mathrm{H}, \mathrm{SH}), 2.29\left(\mathrm{~s}, 3 \mathrm{H}, \mathrm{CH}_{3}\right)$.

${ }^{13} \mathrm{C}$ NMR (75 MHz, $\mathrm{CDCl}_{3}$ ): $\delta=135.6$ (Ar-C4), 129.9 (Ar-C3), 129.8 (Ar$\mathrm{C} 2), 126.6$ ( $\mathrm{Ar}-\mathrm{C} 1), 20.9\left(\mathrm{CH}_{3}\right)$.

\section{2-Methylbenzenethiol (3c) ${ }^{45}$}

Compound 2c (19.93 g, $100 \mathrm{mmol}$ ) was reacted with $\mathrm{Na}_{2} \mathrm{~S} \cdot 10 \mathrm{H}_{2} \mathrm{O}$ (28.8 g, $120 \mathrm{mmol})$.

Yield: $12.30 \mathrm{~g}$ (99\%); colorless liquid; bp $195^{\circ} \mathrm{C}$.

${ }^{1} \mathrm{H}$ NMR $\left(250 \mathrm{MHz}, \mathrm{CDCl}_{3}\right): \delta$ = 7.3-6.9 (m, $\left.4 \mathrm{H}, \mathrm{ArH}\right), 3.17$ (s, $\left.1 \mathrm{H}, \mathrm{SH}\right)$, $2.23\left(\mathrm{~s}, 3 \mathrm{H}, \mathrm{CH}_{3}\right)$.

${ }^{13} \mathrm{C} \mathrm{NMR}\left(75 \mathrm{MHz}, \mathrm{CDCl}_{3}\right): \delta=136.2(\mathrm{Ar}-\mathrm{C} 2), 131.2(\mathrm{Ar}-\mathrm{C} 1)$, $130.5(\mathrm{Ar}-$ C5), 130.1 (Ar-C6), 126.8 (Ar-C4), 126.1 (Ar-C3), $21.2\left(\mathrm{CH}_{3}\right)$.

\section{4-Mercaptoanisole (3d) $)^{45}$}

Compound 2d (21.23 g, $100 \mathrm{mmol})$ was reacted with $\mathrm{Na}_{2} \mathrm{~S} \cdot 10 \mathrm{H}_{2} \mathrm{O}$ (28.8 g, $120 \mathrm{mmol})$.

Yield: $13.60 \mathrm{~g}$ (99\%); colorless liquid; bp $100-103^{\circ} \mathrm{C} / 13 \mathrm{mmHg}$. ${ }^{1} \mathrm{H} \mathrm{NMR}\left(250 \mathrm{MHz}, \mathrm{CDCl}_{3}\right): \delta=7.17\left(\mathrm{~d},{ }^{3} \mathrm{~J}_{\mathrm{H}-\mathrm{H}}=8.7 \mathrm{~Hz}, 2 \mathrm{H}, \mathrm{Ar}-\mathrm{H} 3\right), 6.72$ $\left(\mathrm{d},{ }^{3} J_{\mathrm{H}-\mathrm{H}}=8.7 \mathrm{~Hz}, 2 \mathrm{H}, \mathrm{Ar}-\mathrm{H} 2\right), 3.65\left(\mathrm{~s}, 3 \mathrm{H}, \mathrm{CH}_{3}\right), 3.35(\mathrm{~s}, 1 \mathrm{H}, \mathrm{SH})$. ${ }^{13} \mathrm{C} \mathrm{NMR}\left(75 \mathrm{MHz}, \mathrm{CDCl}_{3}\right.$ ): $\delta=158.5$ (Ar-C4), 132.3 (Ar-C2), 119.9 (ArC1), 114.7 ( $\mathrm{Ar}-\mathrm{C} 3), 55.2\left(\mathrm{CH}_{3}\right)$. 
2-Mercaptoanisole (3e) $)^{45}$

Compound 2 e $(21.23 \mathrm{~g}, 100 \mathrm{mmol})$ was reacted with $\mathrm{Na}_{2} \mathrm{~S} \cdot 10 \mathrm{H}_{2} \mathrm{O}$ $(28.8 \mathrm{~g}, 120 \mathrm{mmol})$.

Yield: $13.88 \mathrm{~g}$ (99\%); colorless liquid; bp $99^{\circ} \mathrm{C} / 8 \mathrm{mmHg}$.

${ }^{1} \mathrm{H}$ NMR $\left(250 \mathrm{MHz}, \mathrm{CDCl}_{3}\right): \delta=7.3-6.7(\mathrm{~m}, 4 \mathrm{H}, \mathrm{ArH}), 3.76(\mathrm{~s}, 1 \mathrm{H}, \mathrm{SH})$, $3.73\left(\mathrm{~s}, 3 \mathrm{H}, \mathrm{CH}_{3}\right)$.

${ }^{13} \mathrm{C}$ NMR $\left(75 \mathrm{MHz}, \mathrm{CDCl}_{3}\right.$ ): $\delta=154.8(\mathrm{Ar}-\mathrm{C} 2), 129.3(\mathrm{Ar}-\mathrm{C} 6), 126.3(\mathrm{Ar}-$ C4), 121.1 (Ar-C5), 120.5 (Ar-C1), 110.7 (Ar-C3), $55.7\left(\mathrm{CH}_{3}\right)$.

\section{3-(Trifluoromethyl)benzenethiol (3f) ${ }^{46}$}

Compound 2 f $(25.02 \mathrm{~g}, 100 \mathrm{mmol})$ was reacted with $\mathrm{Na}_{2} \mathrm{~S} \cdot 10 \mathrm{H}_{2} \mathrm{O}$ (28.8 g, $120 \mathrm{mmol})$.

Yield: $14.43 \mathrm{~g}(81 \%)$; colorless liquid; bp $161-163^{\circ} \mathrm{C}$.

${ }^{1} \mathrm{H}$ NMR (250 MHz, $\left.\mathrm{CDCl}_{3}\right): \delta=7.8-7.0(\mathrm{~m}, 4 \mathrm{H}, \mathrm{ArH}), 3.50(\mathrm{~s}, 1 \mathrm{H}, \mathrm{SH})$.

${ }^{13} \mathrm{C}$ NMR $\left(75 \mathrm{MHz}, \mathrm{CDCl}_{3}\right): \delta=132.7(\mathrm{Ar}-\mathrm{C} 1), 132.4\left(\mathrm{q},{ }^{4} J_{\mathrm{C}-\mathrm{F}}=1.3 \mathrm{~Hz}\right.$, $\mathrm{Ar}-\mathrm{C} 5), 131.6\left(\mathrm{q},{ }^{2} J_{\mathrm{C}-\mathrm{F}}=32.4 \mathrm{~Hz}, \mathrm{Ar}-\mathrm{C} 3\right), 129.4(\mathrm{Ar}-\mathrm{C} 6), 125.8\left(\mathrm{q},{ }^{3} J_{\mathrm{C}-\mathrm{F}}=\right.$ $3.9 \mathrm{~Hz}, \mathrm{Ar}-\mathrm{C} 4), 123.9\left(\mathrm{q},{ }^{1} J_{\mathrm{C}-\mathrm{F}}=272.5 \mathrm{~Hz}, \mathrm{CF}_{3}\right), 122.4\left(\mathrm{q},{ }^{3} J_{\mathrm{C}-\mathrm{F}}=3.9 \mathrm{~Hz}\right.$, Ar-C2).

\section{Naphthalene-1-thiol (3g) ${ }^{47}$}

Compound $2 \mathrm{~g}$ (23.23 g, $100 \mathrm{mmol}$ ) was reacted with $\mathrm{Na}_{2} \mathrm{~S} \cdot 10 \mathrm{H}_{2} \mathrm{O}$ $(28.8 \mathrm{~g}, 120 \mathrm{mmol})$.

Yield: $14.74 \mathrm{~g}$ (92\%); colorless liquid; bp $160-162{ }^{\circ} \mathrm{C} / 20 \mathrm{mmHg}$.

${ }^{1} \mathrm{H}$ NMR $\left(250 \mathrm{MHz}, \mathrm{CDCl}_{3}\right): \delta=8.2-8.0(\mathrm{~m}, 1 \mathrm{H}, \mathrm{Ar}-\mathrm{H} 8), 7.9-6.9(\mathrm{~m}$, $6 \mathrm{H}, \mathrm{ArH}), 3.40$ (s, $1 \mathrm{H}, \mathrm{SH})$.

${ }^{13} \mathrm{C}$ NMR $\left(75 \mathrm{MHz}, \mathrm{CDCl}_{3}\right): \delta=134.2$ (Ar-C9), 132.5 (Ar-C10), 128.9 (Ar-C5), 128.7 (Ar-C1), 128.4 (Ar-C6), 127.3 (Ar-C2), 126.7 (Ar-C7), 126.3 (Ar-C4), 125.9 (Ar-C8), 125.4 (Ar-C3).

\section{Preparation of Diaryl Disulfides 4a-g; General Procedure}

\section{Method A}

A solution of 3-(arylthio)propionic acid (10 mmol) in $2.5 \mathrm{M} \mathrm{NaOH}$ (60 $\mathrm{mL}$ ) was heated at reflux for $1 \mathrm{~h}$. The reaction mixture was then cooled to $0{ }^{\circ} \mathrm{C}$ and finely powdered iodine $(1.52 \mathrm{~g}, 6 \mathrm{mmol})$ was added in portions at a rate such that the inner temperature of the reaction mixture was maintained below $10{ }^{\circ} \mathrm{C}$. The mixture was acidified with cc $\mathrm{HCl}$ to $\mathrm{pH} 1$ and a small amount of sat. $\mathrm{Na}_{2} \mathrm{~S}_{2} \mathrm{O}_{5}$ was added. The solution was extracted with $\mathrm{CHCl}_{3}(3 \times 20 \mathrm{~mL})$, the combined organic phases were washed with $10 \% \mathrm{KHCO}_{3}(20 \mathrm{~mL})$ and water $(20 \mathrm{~mL})$, and was dried over $\mathrm{Na}_{2} \mathrm{SO}_{4}$. The solvent was evaporated and the crude product was purified by crystallization from petroleum ether.

\section{Diphenyl Disulfide (4a) ${ }^{38}$}

3-(Phenylthio)propionic acid 2a (1.82 g, $10 \mathrm{mmol}$ ) was reacted with iodine (1.52 g, $6 \mathrm{mmol})$.

Yield: $1.02 \mathrm{~g}(94 \%)$; crystalline material; $\mathrm{mp} 59-60{ }^{\circ} \mathrm{C}$.

${ }^{1} \mathrm{H}$ NMR $\left(250 \mathrm{MHz}, \mathrm{CDCl}_{3}\right)$ : $\delta=7.6-7.1(\mathrm{~m}, 5 \mathrm{H}, \mathrm{ArH})$,

${ }^{13} \mathrm{C}$ NMR $\left(75 \mathrm{MHz}, \mathrm{CDCl}_{3}\right): \delta=136.9$ (Ar-C1), 128.9 (Ar-C2), $127.5(\mathrm{Ar}-$ C3), 127.0 (Ar-C4).

\section{Di(p-tolyl) Disulfide (4b) $)^{39}$}

3-(p-Tolylthio)propionic acid $\mathbf{2 b}$ (1.99 g, $10 \mathrm{mmol}$ ) was reacted with iodine ( $1.52 \mathrm{~g}, 6 \mathrm{mmol})$.
Yield: $1.21 \mathrm{~g}$ (98\%); crystalline material; $\mathrm{mp} 43-45^{\circ} \mathrm{C}$.

${ }^{1} \mathrm{H}$ NMR $\left(250 \mathrm{MHz}, \mathrm{CDCl}_{3}\right): \delta=7.35\left(\mathrm{~d},{ }^{3} \mathrm{~J}_{\mathrm{H}-\mathrm{H}}=8.3 \mathrm{~Hz}, 2 \mathrm{H}, \mathrm{Ar}-\mathrm{H} 2\right), 7.06$ (d, $\left.{ }^{3} \mathrm{H}_{\mathrm{H}-\mathrm{H}}=8.3 \mathrm{~Hz}, 2 \mathrm{H}, \mathrm{Ar}-\mathrm{H} 3\right), 2.28\left(\mathrm{~s}, 3 \mathrm{H}, \mathrm{CH}_{3}\right)$.

${ }^{13} \mathrm{C} \mathrm{NMR}\left(75 \mathrm{MHz}, \mathrm{CDCl}_{3}\right.$ ): $\delta=137.4$ (Ar-C4), 133.9 (Ar-C1), 129.8 (ArC2), 128.5 (Ar-C3), $21.0\left(\mathrm{CH}_{3}\right)$.

\section{Di(o-tolyl) Disulfide (4c) $)^{39}$}

3-(o-Tolylthio)propionic acid 2c (1.99 g, $10 \mathrm{mmol})$ was reacted with iodine ( $1.52 \mathrm{~g}, 6 \mathrm{mmol})$.

Yield: $1.17 \mathrm{~g}$ (95\%); crystalline material; $\mathrm{mp} 30-34{ }^{\circ} \mathrm{C}$.

${ }^{1} \mathrm{H}$ NMR (250 MHz, $\left.\mathrm{CDCl}_{3}\right): \delta=7.5-7.0(\mathrm{~m}, 4 \mathrm{H}, \mathrm{ArH}), 2.39(\mathrm{~s}, 3 \mathrm{H}$, $\mathrm{CH}_{3}$ ).

${ }^{13} \mathrm{C}$ NMR $\left(75 \mathrm{MHz}, \mathrm{CDCl}_{3}\right)$ : $\delta=137.4(\mathrm{Ar}-\mathrm{C} 2), 135.4(\mathrm{Ar}-\mathrm{C} 1), 130.3(\mathrm{Ar}-$ C5), 128.7 (Ar-C6), 127.3 (Ar-C4), 126.7 (Ar-C3), $20.0\left(\mathrm{CH}_{3}\right)$.

\section{Di(4-methoxyphenyl) Disulfide (4d) $)^{39}$}

3-((4-Methoxyphenyl)thio)propionic acid 2d (2.12 g, $10 \mathrm{mmol})$ was reacted with iodine $(1.52 \mathrm{~g}, 6 \mathrm{mmol})$.

Yield: $0.90 \mathrm{~g}(65 \%)$; crystalline material; $\mathrm{mp} 35-37^{\circ} \mathrm{C}$.

${ }^{1} \mathrm{H} \mathrm{NMR}\left(250 \mathrm{MHz}, \mathrm{CDCl}_{3}\right): \delta=7.35\left(\mathrm{~d},{ }^{3} J_{\mathrm{H}-\mathrm{H}}=8.7 \mathrm{~Hz}, 2 \mathrm{H}, \mathrm{Ar}-\mathrm{H} 3\right), 6.81$ (d, $\left.{ }^{3} J_{\mathrm{H}-\mathrm{H}}=8.7 \mathrm{~Hz}, 2 \mathrm{H}, \mathrm{Ar}-\mathrm{H} 2\right), 3.74\left(\mathrm{~s}, 3 \mathrm{H}, \mathrm{CH}_{3}\right)$.

${ }^{13} \mathrm{C} \mathrm{NMR}\left(75 \mathrm{MHz}, \mathrm{CDCl}_{3}\right.$ ): $\delta=159.0(\mathrm{Ar}-\mathrm{C} 4), 132.8$ (Ar-C2), $127.4(\mathrm{Ar}-$ C1), 114.7 (Ar-C3), $55.4\left(\mathrm{CH}_{3}\right)$.

\section{Di(2-methoxyphenyl) Disulfide (4e) ${ }^{40}$}

3-((2-Methoxyphenyl)thio)propionic acid 2 e (2.12 g, $10 \mathrm{mmol})$ was reacted with iodine ( $1.52 \mathrm{~g}, 6 \mathrm{mmol})$.

Yield: $1.23 \mathrm{~g}(88 \%)$; crystalline material; $\mathrm{mp} 119-121^{\circ} \mathrm{C}$.

${ }^{1} \mathrm{H}$ NMR (250 MHz, $\left.\mathrm{CDCl}_{3}\right): \delta=7.5-6.9(\mathrm{~m}, 4 \mathrm{H}, \operatorname{ArH}), 3.87(\mathrm{~s}, 3 \mathrm{H}$, $\mathrm{CH}_{3}$ ).

${ }^{13} \mathrm{C} \mathrm{NMR}\left(75 \mathrm{MHz}, \mathrm{CDCl}_{3}\right)$ : $\delta=156.4(\mathrm{Ar}-\mathrm{C} 2), 137.6$ (Ar-C6), $127.3(\mathrm{Ar}-$ C4), 124.2 (Ar-C5), 121.1 (Ar-C1), $110.3(\mathrm{Ar}-\mathrm{C} 3), 55.6\left(\mathrm{CH}_{3}\right)$.

Bis(3-(trifluoromethyl)phenyl) Disulfide (4f) ${ }^{32}$

3-((3-(Trifluoromethyl)phenyl)thio)propionic acid 2f (2.50 g, 10 $\mathrm{mmol}$ ) was reacted with iodine (1.52 g, $6 \mathrm{mmol})$.

Yield: $1.56 \mathrm{~g}$ (88\%); crystalline material; bp $95-100^{\circ} \mathrm{C} / 0.2 \mathrm{mmHg}$.

${ }^{1} \mathrm{H}$ NMR $\left(250 \mathrm{MHz}, \mathrm{CDCl}_{3}\right): \delta=7.8-7.1(\mathrm{~m}, 4 \mathrm{H}, \mathrm{ArH})$.

${ }^{13} \mathrm{C}$ NMR $\left(75 \mathrm{MHz}, \mathrm{CDCl}_{3}\right): \delta=138.0(\mathrm{Ar}-\mathrm{C} 1), 131.9\left(\mathrm{q},{ }^{2} J_{\mathrm{C}-\mathrm{F}}=32.5 \mathrm{~Hz}\right.$, Ar-C3), $130.9\left(\mathrm{q}, \mathrm{J}_{\mathrm{C}-\mathrm{F}}=1.2 \mathrm{~Hz}, \mathrm{Ar}-\mathrm{C} 5\right), 129.8(\mathrm{Ar}-\mathrm{C} 6), 124.5\left(\mathrm{q}, \mathrm{J}_{\mathrm{C}-\mathrm{F}}=\right.$ $3.9 \mathrm{~Hz}, \mathrm{Ar}-\mathrm{C} 2), 124.4\left(\mathrm{q},{ }^{3} J_{\mathrm{C}-\mathrm{F}}=3.9 \mathrm{~Hz}, \mathrm{Ar}-\mathrm{C} 4\right), 123.8\left(\mathrm{q},{ }^{1} J_{\mathrm{C}-\mathrm{F}}=272.5 \mathrm{~Hz}\right.$, $\left.\mathrm{CF}_{3}\right)$.

\section{Di(naphthalen-1-yl) Disulfide (4g) $)^{39}$}

3-((Naphthalen-1-yl)thio)propionic acid $\mathbf{2 g}$ (2.32 g, $10 \mathrm{mmol})$ was reacted with iodine (1.52 g, $6 \mathrm{mmol}$ ).

Yield: $1.50 \mathrm{~g}(94 \%)$; crystalline material; $\mathrm{mp} 83-86{ }^{\circ} \mathrm{C}$.

${ }^{1} \mathrm{H}$ NMR (250 MHz, $\left.\mathrm{CDCl}_{3}\right): \delta=8.4-8.1(\mathrm{~m}, 1 \mathrm{H}, \mathrm{Ar}-\mathrm{H} 8), 7.9-7.1(\mathrm{~m}$, $6 \mathrm{H}, \mathrm{ArH})$.

${ }^{13} \mathrm{C}$ NMR (75 MHz, $\mathrm{CDCl}_{3}$ ): $\delta=134.1$ (Ar-C9), 132.6 (Ar-C10), 130.3 (Ar-C5), 129.9 (Ar-C1), 128.6 (Ar-C6), 128.0 (Ar-C2), 126.8 (Ar-C7), 126.5 (Ar-C4), 125.9 (Ar-C8), 125.1 (Ar-C3). 


\section{Method B}

To a solution of arylmercaptan $(20 \mathrm{mmol})$ in absolute pyridine (10 $\mathrm{mL})$ a solution of PTAB $(3.95 \mathrm{~g}, 10.5 \mathrm{mmol})$ in absolute pyridine (10 $\mathrm{mL}$ ) was added dropwise. The unreacted reagent was neutralized with sat. $\mathrm{NaHSO}_{3}$, and the mixture was poured into a mixture of ice $(100 \mathrm{~g})$ and $6 \mathrm{M} \mathrm{HCl}(50 \mathrm{~mL})$. The solution was extracted with $\mathrm{CHCl}_{3}$ $(3 \times 40 \mathrm{~mL})$, the combined organic phases were washed with $10 \%$ $\mathrm{KHCO}_{3}(40 \mathrm{~mL})$ and water $(40 \mathrm{~mL})$, and was dried over $\mathrm{Na}_{2} \mathrm{SO}_{4}$. The solvent was evaporated and the crude product was purified by crystallization from petroleum ether. The physical and spectral properties of the product were identical to those obtained by Method A.

\section{Diphenyl Disulfide (4a) ${ }^{38}$}

Thiophenol 3a (2.05 mL, $2.20 \mathrm{~g}, 20 \mathrm{mmol})$ was reacted with PTAB (3.9 g, $10.5 \mathrm{mmol})$.

Yield: $1.92 \mathrm{~g}(88 \%)$.

The physical and spectral properties of the product are identical to those obtained by Method A.

\section{Di(p-tolyl) Disulfide (4b) $)^{39}$}

4-Methylbenzenethiol $\mathbf{3 b}(2.48 \mathrm{~g}, 20 \mathrm{mmol})$ was reacted with PTAB (3.9 g, $10.5 \mathrm{mmol})$.

Yield: $1.31 \mathrm{~g}$ (53\%).

The physical and spectral properties of the product are identical to those obtained by Method A.

\section{Di(o-tolyl) Disulfide (4c) ${ }^{39}$}

2-Methylbenzenethiol 3c (2.35 mL, $2.48 \mathrm{~g}, 20 \mathrm{mmol})$ was reacted with PTAB (3.9 g, $10.5 \mathrm{mmol})$.

Yield: $1.50 \mathrm{~g}$ (61\%).

The physical and spectral properties of the product are identical to those obtained by Method A.

\section{Di(4-methoxyphenyl) Disulfide (4d) ${ }^{39}$}

4-Mercaptoanizole 3d (2.46 mL, $2.80 \mathrm{~g}, 20 \mathrm{mmol})$ was reacted with $\operatorname{PTAB}(3.9 \mathrm{~g}, 10.5 \mathrm{mmol})$.

Yield: $2.17 \mathrm{~g}$ (78\%).

The physical and spectral properties of the product are identical to those obtained by Method A.

\section{Di(2-methoxyphenyl) Disulfide (4e) ${ }^{40}$}

2-Mercaptoanizole $3 e(2.43 \mathrm{~mL}, 2.80 \mathrm{~g}, 20 \mathrm{mmol})$ was reacted with $\operatorname{PTAB}(3.9 \mathrm{~g}, 10.5 \mathrm{mmol})$.

Yield: $1.75 \mathrm{~g}$ (63\%).

The physical and spectral properties of the product are identical to those obtained by Method A.

\section{Bis(3-(trifluoromethyl)phenyl) Disulfide (4f) $)^{41}$}

3-(Trifluoromethyl)benzenethiol 3f (3.56 g, $20 \mathrm{mmol}$ ) was reacted with PTAB (3.9 g, $10.5 \mathrm{mmol}$ ).

Yield: $1.98 \mathrm{~g}$ (56\%).

The physical and spectral properties of the product are identical to those obtained by Method A.

\section{Di(naphthalen-1-yl) Disulfide (4g) ${ }^{39}$}

Naphthalene-1-thiol $3 \mathrm{~g}$ (2.77 mL, $3.21 \mathrm{~g}, 20 \mathrm{mmol})$ was reacted with $\operatorname{PTAB}$ (3.9 g, $10.5 \mathrm{mmol}$ ).

Yield: $1.69 \mathrm{~g}$ (53\%).

\section{Funding Information}

National Research Development and Innovation Office (NN 117633).

\section{Acknowledgment}

The authors thank Dr. H. Medzihradszky-Schweiger for SH analyses.

\section{Supporting Information}

Supporting information for this article is available online at https://doi.org/10.1055/s-0036-1591771.

\section{References}

(1) Bingul, M.; Tan, O.; Gardner, C. R.; Sutton, S. K.; Arndt, G. M.; Marshall, G. M.; Cheung, B. B.; Kumar, N.; Black, D. St. C. Molecules 2016, 21, 916.

(2) Okaecwe, T.; Swanepoel, A. J.; Petzer, A.; Bergh, J. J.; Petzer, J. P. Bioorg. Med. Chem. 2012, 20, 4336.

(3) Jia, W.; Liu, Y.; Li, W.; Liu, Y.; Zhang, D.; Zhang, P.; Gong, P. Bioorg. Med. Chem. 2009, 17, 4569.

(4) Siegl, P. K. S.; Goldberg, A. I.; Goldberg, M. R.; Chang, P. I. US. Pat. 5817658, 06 Oct, 1998.

(5) Frank, R. PCT Int. Appl 2006122771, 23 Nov, 2006.

(6) Lynch, J. J. Jr.; Salata, J. J. PCT Int. Appl 9800405, 08 Jan, 1998.

(7) (a) Gao, S.; Tseng, C.; Tsai, C. H. Yao C.-F. Tetrahedron 2008, 64, 1955. (b) Petropoulos, J. C.; McCall, M. A.; Tarbell, D. S. J. Am. Chem. Soc. 1953, 75, 1130. (c) Arndt, F.; Loeve, L.; Ayca, E. Chem. Ber. 1954, 84, 329. (d) Kresze, G.; Schramm, W.; Cleve, G. Chem. Ber. 1961, 94, 2060.

(8) (a) Arndt, F.; Flemming, W.; Scholz, E.; Löwensohn, V. Ber. Dtsch. Chem. Ges. 1923, 56, 1269. (b) Krollpfeiffer, F.; Schultze, H. Ber. Dtsch. Chem. Ges. 1923, 56, 1819. (c) Gresham, T. L.; Jansen, J. E.; Shaver, F. W.; Bankert, R. A.; Beears, W. L.; Predengast, M. G. J. Am. Chem. Soc. 1949, 71, 661. (d) Sen, A. B.; Arora, S. L. J. Indian Chem. Soc. 1958, 35, 197. (e) Node, M.; Nishide, K.; Ochiai, M.; Fuji, K.; Fujita, E. J. Org. Chem. 1981, 46, 5163.

(9) Hurd, C. D.; Hayao, S. J. Am. Chem. Soc. 1954, 76, 5065.

(10) Gogia, S.; Sirohi, R.; Gupta, S.; Kishore, D.; Joshi, B. C. J. Indian Chem. Soc. 2004, 81, 515.

(11) Becht, J.-M.; Wagner, A.; Mioskowski, C. J. Org. Chem. 2003, 68, 5758.

(12) (a) Jepsen, T. H.; Larsen, M.; Jørgensen, M.; Nielsen, M. B. Tetrahedron Lett. 2011, 52, 4045. (b) Itoh, T.; Mase, T. Org. Lett. 2004, 6, 4587.

(13) Wang, P.; Zhang, J.; He, H.; Jin, Y. Nanoscale 2014, 6, 13470.

(14) Jadzinsky, P. D.; Calero, G.; Ackerson, C. J.; Bushnell, D. A.; Kornberg, R. D. Science 2007, 318, 430.

(15) Xu, M.; Lu, N.; Qi, D.; Xu, H.; Wang, Y.; Shi, S.; Chi, L. J. Colloid Interface Sci. 2011, 360, 300.

(16) Bindoli, A.; Fukuto, J. M.; Forman, H. J. Antioxid. Redox Signal. 2008, 10, 1549. 
(17) Mahmood, N.; Jhaumeer-Lauloo, S.; Sampson, J.; Houghton, P. J. J. Pharm. Pharmacol. 1998, 50, 1339.

(18) (a) Allen, C. F. H.; MacKay, D. D. Org. Synth. 1932, 12, 76. (b) Bhaumik, I.; Misra, A. K. SynOpen 2017, 1, 117.

(19) Kuhle, E. The Chemistry of the Sulfenic Acids; Georg Thieme: Stuttgart, 1979.

(20) Douglass, I. B. J. Org. Chem. 1974, 39, 563.

(21) Youn, J.-H.; Herrmann, R. Tetrahedron Lett. 1986, 27, 1493.

(22) Nishiyama, Y.; Kawamatsu, H.; Sonoda, N. J. Org. Chem. 2005, 70, 2551.

(23) Smid, T.; Blees, J. S.; Bajer, M. M.; Wild, J.; Pescatori, L.; Crucitti, G. C.; Scipione, L.; Costi, R.; Heinrich, C. J.; Brüne, B.; Colburn, N. H.; Di Santo, R. PLoS ONE 2016, 11, e0151643.

(24) Rice, W. G.; Turpin, J. A.; Schaffer, C. A.; Graham, L.; Clanton, D.; Buckheit, R. W. Jr.; Zaharevitz, D.; Summers, A.; Wallqvist, A.; Corell, D. G. J. Med. Chem. 1996, 39, 3606.

(25) Gundermann, K.-D.; Hümke, K. In Houben-Weyl; E 11/1, Georg Thieme Verlag: Stuttgart, 1985, 32.

(26) (a) Gundermann, K.-D.; Hümke, K. In Houben-Weyl; E 11/1, Georg Thieme Verlag: Stuttgart, 1985, p. 129. (b) Suzuki, H.; Shinoda, M. Bull. Chem. Soc. Jpn. 1977, 50, 321. (c) Drabowitz, J.; Mikołajczik, M. Synthesis 1980, 32. (d) Dhar, P.; Ranjan, R.; Chandrsekaran, S. J. Org. Chem. 1990, 55, 3728. (e) Sato, T.; Otera, J.; Nozaki, H. Tetrahedron Lett. 1990, 31, 3591.

(27) Osuka, A.; Ohmasa, K.; Uno, Y.; Suzuki, H. Synthesis 1983, 68.

(28) Zhang, Z.; Zhou, X.; Xie, Y.; Greenberg, M. M.; Xi, Z.; Zhou, C. J. Am. Chem. Soc. 2017, 139, 6146.

(29) Baldwin, A. D.; Kiick, K. L. Bioconjugate Chem. 2011, 22, 1946.
(30) Weismann, M. R.; Winger, K. T.; Ghiassan, S.; Gobbo, P.; Workentin, M. S. Bioconjugate Chem. 2016, 27, 586.

(31) Holmberg, B.; Schjånberg, E. Ark. Kemi. Mineral. Geol. 1942, A15, No. 20; Chem. Abstr. 1944, 38: 2943; Chem. Zentralb.; 1943, (I), 388.

(32) Rábai, J. Synthesis 1989, 523.

(33) Krollpfeiffer, F.; Schultze, H.; Schlumbohm, E.; Sommermeyer, E. Ber. Dtsch. Chem. Ges. 1925, 58, 1654.

(34) Jacquignon, P.; Fravolini, A.; Feron, A.; Croisy, A. Experimentia 1974, 30, 452.

(35) Kreevoy, M. M.; Harper, E. T.; Duvall, R. E.; Wilgus III, H. S.; Ditsch, L. T. J. Am. Chem. Soc. 1960, 82, 4899.

(36) Dean, R. T.; Hook, E. O. US Patent 2,450,634 (1942, Am. Cyanamid Co.); Chem. Abstr. 1949, 895.

(37) Otto, R.; Tröger, J. Ber. Dtsch. Chem. Ges. 1891, 24, 1145.

(38) Spyroudis, S.; Varvoglis, A. Synthesis 1975, 445.

(39) Vinkler, E.; Klivényi, F. Acta Chim. Acad. Sci. Hung. 1954, 5, 159.

(40) Gattermann, L. Ber. Dtsch. Chem. Ges. 1899, 32, 1136.

(41) Reich, H. J.; Willis, Jr. W. W.; Clark, P. D. J. Org. Chem. 1981, 46, 2775.

(42) Rábai, J.; Kapovits, I.; Tanács, B.; Tamás, J. Synthesis 1990, 847.

(43) Morris, J. C.; Lanum, W. J.; Helm, R. V.; Haines, W. E.; Cook, G. L.; Ball, J. S. J. Chem. Eng. Data 1960, 5, 112.

(44) Kharash, N.; Swidler, R. J. Org. Chem. 1954, 19, 1704.

(45) Cabiddu, S.; Melis, S.; Piras, P. P.; Sotgiu, F. Synthesis 1982, 583.

(46) Mindl, J.; Balcárek, P.; Šilar, L.; Večeřa, M. Collect. Czech. Chem. Commun. 1980, 45, 3130.

(47) Grillot, G. F.; Levin, P. M.; Green, R.; Ashford, R. B. J. Am. Chem. Soc. 1950, 72, 1863. 